BULL. AUSTRAL. MATH. SOC.

VOL. 13 (1975), 467-468.

\title{
On the structure of geometries with spinor-type connexion
}

\section{M.C. Cullinan}

The aim of this thesis is to present a synthesis, using standard techniques of algebra and of differential geometry, of some of the principal ideas and structures of the classical field-theoretic description of spinor fields in curved space-time. A geometric model for spinor fields in curved space-time is described which is based upon properties of socalled tangent Clifford algebras. The tangent Clifford algebra to a spacetime manifold at a certain point is the quotient space of the algebra of covariant tensors at the point by a certain two-sided ideal, and is uniquely defined once the metric structure of the space-time manifold is given. Minimal left ideals of each tangent Clifford algebra are identified with spaces of four component spinors at the point. This model therefore features a direct method for synthesizing spinor fields from vector and tensor fields on a space-time manifold.

Since a spanning set for each tangent Clifford algebra necessarily involves basis elements of four minimal left ideals, one is led to associate four four-dimensional spin-spaces with each point of a space-time manifold. The possibility of making transformations between these four spin spaces at each point offers a simple geometrical model for the class of unitary symmetry theories which relate to symmetries over a quartet of four dimensional spin spaces. Fields of higher spin are also readily accommodated in this model; as with the four-component spinor objects mentioned earlier, geometrical concomitants of (spinor-) fields of higher spin are constructed entirely from vector and tensor fields of various kinds.

Received 8 July 1975. Thesis submitted to the University of New South Wales, March 1975. Degree approved, August 1975. Supervisor: Professor G. Szekeres. 
A theory of connexion is proposed for the spinor objects described above; this theory uses only standard methods of classical differential geometry, together with a certain amount of algebra, and leads, amongst other things, to the realization of a rather explicit relationship between the operation of taking the covariant differential of a tensor quantity on the one hand, and that of taking a 'Dirac'-type derivative $\left.\underline{\Omega}^{\phi}(), \phi\right)$ of associated spinor quantities on the other. This theory is more general than existing models for spinor geometry as it embraces not only the usual theory of spinor connexion, but also a geometrical model for a certain class of generalized (Yang-Mills type) gauge fields in curved space-time. of particular interest in this context are those space-time manifolds which do not admit a so-called spinor structure, for on these manifolds a geometry of spinors will generally involve connexion quantities which are direct geometrical concomitants of gauge fields such as the isotopic gauge field of Yang and Mills.

At each stage in the development of this model, explicit calculations are given which serve to relate the various results to those of standard physical theory. In particular, a description of the so-called generalized Dirac matrices which embodies both algebraic and differential properties is obtained from the general structure of the model. Like the other spinor objects with which this model deals, these matrices and their properties derive entirely from space-time properties of vector and tensor fields. 